\title{
Etude du régime alimentaire des gardons (Rutilus rutilus (L.) ) du lac de Pareloup (Massif Central, France)
}

\author{
C. Richeux ${ }^{1}$ \\ J.E. Arias-Gonzalez 2 \\ J.N. Tourenq ${ }^{1}$
}

Mots clés : Poissons, Rutilus ruilus, régime alimentaire, contenus intestinaux.

Les préférences alimentaires des gardons du lac de Pareloup ont été mises en évidence par l'analyse de leurs contenus intestinaux de janvier à juillet 1990. Les spectres alimentaires ont été établis grâce à l'indice de poids qui a permis de visualiser une hiérarchie des proies en fonction de la classe de taille et de la saison. Ces spectres montrent que les gardons de 10 à $21 \mathrm{~cm}$ ont une alimentation à base de détritus et de zooplancton alors que les individus plus âgés sont détritivores. Il existe une variation saisonnière du régime des gardions : les détritus dominent en hiver ; ils sont progressivement remplacés par le zooplancton (Cladocères) et, en quantité moindre, par les macroinvertébrés (Chironomidae) à partir de mars.

Le phytoplancton (Diatomophycées) est ingéré en grande quantité, mais sa contribution pondérale est toujours faible. Les gardons de Pareloup se nourrissent activement pendant toute la période d'étude et de façon assez opportuniste.

\section{A study of the diet of roach (Rutilus rutilus (L.)) in the lake of Pareloup (Massif Central, France)}

Keywords : Fish, Rutilus rutilus, diet, gut contents.

The diet preferences of roach in the lake of Pareloup have been determined by analyses of their gut contents from January to July 1990 . Changes in the food composition have been established by using a weight index that illustrates a hierarchy of prey in relation to the season. These analyses show that roach in the size range $10-21 \mathrm{~cm}$ have a diet of chiefly detritus and zooplankton whilst the oldest individuals are detritivores. There is a seasonal variation in the roach diet : detritus dominates in winter, it is replaced progressively by zooplankton (Cladocera) and, in lesser quantity, by macroinvertebrates (Chironomidae) from March.

Phytoplankton (diatoms) are ingested in large quantities, but their contribution in terms of weight of food is always small. The roach of Pareloup fed actively throughout the study period and in a rather opportune way.

\section{Introduction}

La régulation des écosystèmes est souvent interprétée comme le résultat de l'influence des niveaux inférieurs de la chaîne trophique sur l'ensemble des communautés qui composent l'écosystème

1. Laboratoire d'Hydrobiologie, URA 695 C.N.R.S., Université Paul-Sabatier, 118, route de Narbonne, F-31062 Toulouse Cedex.

2. Laboratoire d'Ichtyoécologie Tropicale et Méditerranéenne, U.R.A. 1453 du C.N.R.S., Université de Perpignan, F-66860 Perpignan Cedex. J.E. Arias-Gonzalez remercie le C.O.N.A.C.Y.T. (Mexique) et le C.E.F.I.-International pour son soutien financier.
(Northcote 1988). Moins nombreux sont les travaux concernant les influences en sens inverse (Straskraba 1965).

L'étude des régimes alimentaires des poissons s'avère d'un grand intérêt ; elle permet d'estimer l'impact des populations piscicoles sur la structure des communautés de l'écosystème.

Ces données sont utilisées comme source d'informations pour bâtir des modèles d'interactions entre populations (Palomares \& Pauly 1989).

L'étude du régime alimentaire de l'espèce la plus représentée parmi les communautés pisciaires du lac 
de Pareloup Rutilus rutilus (L.), fait partie des nombreuses données limnologiques et hydrobiologiques collectées depuis 1986 pour la mise au point du modèle de fonctionnement de cet écosystème.

Cette étude réalisée entre janvier et juillet 1990 avait pour but de mettre en évidence les variations intraspécifiques du régime alimentaire en fonction de la taille des poissons et les variations saisonnières de leur spectre alimentaire en fonction de la nourriture disponible dans le milieu.

\section{Matériel et méthodes}

\subsection{Capture des poissons}

Les pêches ont été effectuées sur le lac de Pareloup, retenue hydroélectrique située dans l'Aveyron (altitude : $850 \mathrm{~m}$, superficie : 1350 ha, profondeur moyenne : $12,50 \mathrm{~m}$, profondeur maximale : $35 \mathrm{~m}$, température moyenne de l'eau : $9^{\circ} \mathrm{C}\left(2{ }^{\circ} \mathrm{C}-20^{\circ} \mathrm{C}\right)$.

Les poissons ont été capturés à l'aide de deux batteries de trois filets maillants de fond (ralingue de base fortement plombée : $40 \mathrm{~g} / \mathrm{m}$ ) de type monofilament, de vide de maille 14,18 , et $27 \mathrm{~mm}$ d'entrenceuds, d'une longueur de $27 \mathrm{~m}$ sur $1,50 \mathrm{~m}$ de haut et disposés perpendiculairement à la berge. Les batteries sont relevées toutes les deux heures sur un cycle de 24 heures afin d'étudier le rythme nycthéméral (Arias et al., sous presse).

Des pêches ont été effectuées tous les deux mois, de janvier à juillet 1990. Les gardons capturés sont immédiatement mesurés ( $\pm 1 \mathrm{~mm}$ ), pesés ( $\pm 1 \mathrm{mg}$ ), sacrifiés et disséqués. Les poissons sont ensuite regroupés en trois classes de tailie (longueurs totales en $\mathrm{cm}$ ) définies comme suit : 10-15,4 (I) ; 15,5-20,9 (II) ; 21-26,4 (III).

La disponibilité en plancton du milieu a été estimée à chaque campagne à différentes profondeurs : tous les $5 \mathrm{~m}$ de la surface au fond. L'échantillonnage est réalisé au niveau des filets (h max. : $15 \mathrm{~m}$ ) à l'aide d'une bouteille de type Van Dorn de 5 I. Les prélèvements sont fixés au formol à $5 \%$.

\subsection{Préparation et analyse des contenus intestinaux}

Les gardons étant dépourvus d'estomac, les analyses ont porté sur l'ensemble du tube digestif, de l'oesophage à la partie anale (Hynes 1950, AlbertineBerhaut 1973, Linfield 1980) et non pas uniquement sur le premier tiers du tube comme le suggèrent certains auteurs (Ponton \& Gerdeaux 1988) en raison de la digestion moins avancée dans cette partie.

Les tubes digestifs sont récupérés dans $10 \mathrm{ml}$ de formol à $5 \%$ et leurs contenus pesés ultérieurement $( \pm 100 \mu \mathrm{g}$ ).

Une quinzaine d'individus ( 5 par classe de taille) possèdant les poids frais de contenus intestinaux les plus élevés ont été sélectionnés par campagne de pêche (en écartant délibérément ceux dont les sucs digestifs contribuaient visiblement trop au poids frais) pour une analyse individuelle qualitative et quantitative.

Les macroinvertébrés sont identifiés (au genre, à la famille ou au groupe écologique selon l'état de digestion), dénombrés à la loupe binoculaire, séchés et pesés par taxons.

Le zooplancton est identifié (au niveau générique ou spécifique) et dénombré à la loupe binoculaire après dilution. Les individus sont ensuite mesurés au micromètre oculaire.

Le reste de l'échantillon est filtré sur tamis de $100 \mu \mathrm{m}$ de vide de maille. L'identification générique et le comptage des cellules phytoplanctoniques et des Rotifères ont été effectués sur une fraction du filtrat au microscope inversé (Zeiss Axiovert 10).

Les éléments retenus par le tamis (fraction zooplanctonique et éléments détritiques) ont été séchés et pesés.

Le terme « Autres » désigne les grains de pollen, les spores de champignons dénombrés au microscope inversé mais non pesables, les fourreaux de Trichoptères et les fragments de végétaux macroscopiques. On réserve le terme « Détritus » à l'ensemble des débris minéraux, végétaux et animaux non identifiables et indénombrables.

\subsection{Analyses quantitatives}

Après identification, les items sont regroupés par classes (phytoplancton), familles ou groupes écologiques (zooplancton, macroinvertébrés). Une moyenne est effectuée sur le nombre (N) et le poids (W) par classe de taille et par période d'étude.

Il existe plusieurs méthodes d'analyse et de présentation des données (Hynes 1950, Hyslop 1980). L'indice alimentaire proposé par Lauzanne (1976) : I.A. $=(\% 0 \mathrm{OC} * \% \mathrm{~W}) / 100$ a paru le mieux adapté à la méthode et aux objectifs de l'étude. Il présente l'avantage de tenir compte à la fois des 
préférences alimentaires du prédateur et de l'importance pondérale des proies présentes (Windell 1968, Ponton \& Stroffek 1987).

$$
\begin{gathered}
\text { Nombre d'estomacs renfermant une catégorie de proies } \\
\text { Nombre d'estomacs renfermant de la nourriture } \\
\begin{array}{l}
\mathrm{N} \\
\sum \mathrm{Wij}
\end{array} \\
\begin{array}{r}
\mathrm{N}=1 \\
\mathrm{~J}=\frac{\mathrm{N}}{\mathrm{E} W \mathrm{Wj}} \\
\mathrm{J}=\mathrm{IN}=1
\end{array}
\end{gathered}
$$$$
\% O C=\longrightarrow * 100
$$

Waj $=$ Poids $\sec (e n \mu g)$ d'un individu d'une catégorie de proies.

$\mathrm{J}=$ Nombre total de catégories de proies.

$\mathrm{N}=$ Nombre total d'individus d'une catégorie de proies.

Le plancton d'un volume déterminé compris entre 1,5 et 2 litres par profondeur est concentré dans un flacon de $125 \mathrm{ml}$. Sur ces $125 \mathrm{ml}, 20$ sont prélevés pour étudier le phytoplancton ; le volume restant est filtré sur membrane $0,22 \mu \mathrm{m}$. Le zooplancton ainsi filtré est remis en suspension et conservé dans $10 \mathrm{ml}$ d'alcool à $75 \%$.
Le plancton est compté sous la loupe binoculaire et au microscope inversé. Les quantités (nombre et biomasse) par catégorie de proies sont ramenées au litre pour toute la colonne d'eau prospectée.

Les biomasses zooplanctoniques ont été calculées d'après les relations taille-poids empruntées à la littérature : Bottrell et al. (1976) pour Daphnia Iongispina (O.F. Müller 1785) et Dumont et al. (1975) pour les autres espèces.

$$
\left.W(p g \text { P.S. })=0,47 V^{0,49} \mu^{\prime} N_{\mu} m^{3}=N^{*} V^{\prime}\right)
$$

où $\mathrm{V}^{\prime}=$ Volume (en $\mu \mathrm{m}^{3}$ ) d'une cellule d'un genre considéré.

$N=$ Volume total de cellules du gense consideré.

$V=$ Volume total (en $\left.\mu^{1}\right)$ des cellules du genre considéré.

Les valeurs de $V$ ' résultent de nos mesures (Tableaux 1 et 2) ou ont été empruntées à la littérature (Reynolds 1984, Le Cohu et al., 1991).

Les macroinvertébrés et les détritus ont été séchés à l'étuve à $60^{\circ} \mathrm{C} 24$ heures (obtention du poids constant) avant d'être pesés.

La contribution des items de la catégorie "Autres" au poids total des contenus intestinaux a été considérée en général négligeable vu leur faible masse.

Tableau 1. Biovolumes des principaux genres phytoplanctoniques déterminés.

*** Biovolumes extraits de Reynolds (1984).

** Biovolumes selon Le Cohu et al. (1991).

* Biovolumes moyens (moyenne des genres de la classe ou des genres de morphologie proche).

Table 1. Biovolumes of the main phytoplankton genera.

*** Biovolumes extracted from Reynolds (1984).

\begin{tabular}{|c|c|c|c|c|c|c|c|c|}
\hline GENRES & $V(\mu \mathrm{m} 3)$ & ORIGINE & : GENRES & n3) & ORIGINE & GENRES & $V(u m 3)$ & ORIGINE \\
\hline Achnanthes & 100 & $\star \star$ & Cymbella & 600 & $* *$ & Oocystis & 200 & $\star \star$ \\
\hline Amphiprora & 1124 & $*$ & : Dictyosphaerium & 22,5 & 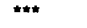 & Oscillatoria & 15 & $\star \star$ \\
\hline Amphora & 600 & * & Dinobryon & 700 & $\star \star \star$ & Phacus & 1200 & $\approx *$ \\
\hline Anabaena & 102 & $\star \star *$ & : Epithemia & 600 & $*$ & Pinnularia & 500 & $\star \star$ \\
\hline Aphanizomenon & 8,2 & * & : Fragilaria & 623 & $\star \star$ & Rhizosolenia & 4000 & 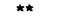 \\
\hline Asterionella & 650 & $\star \star \star$ & Gloeothece Col.50um & 500 & $* *$ & Rhodomonas & 72 & $\star \star \star *$ \\
\hline Ceratium & 45000 & $\star \star$ & Golenkinia & N.D. & $\cdot$ & Scenedesmus & 1000 & $\# *$ \\
\hline Chlamydomonas & 300 & ** & Gomphonema & 1750 & \#* & Sphaerocystis & 600 & $\star * *$ \\
\hline Chodatelia & 250 & $\star$ & : Gymnodinium & $f(\theta)$ & $*$ & Spondylosium & 1300 & $*$ \\
\hline Chryso. Kyste & 600 & $* *$ & : Gyrosigma & 1750 & $\star \star \star$ & : Staurastrum & 20000 & $\pi \pi *$ \\
\hline Closterium & 4520 & $\star \star *$ & : Mallomonas & 1400 & $\star \star$ & : Surirella & 60000 & $\star \star$ \\
\hline Cocconeis & 800 & $\star$ & : Melosira & 593 & **** & : Synedra & 500 & $*$ \\
\hline Cosmarium & 7580 & $m * *$ & : Meridion & 60 & $m$ & : Tabellaria & 1725 & 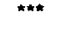 \\
\hline Crucigenia & 200 & $*$ & : Merismopedia & 25 & $*$ & : Tetraedron & 80 & $m *$ \\
\hline Cryptomonas & 2500 & 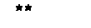 & : Navicula & 1750 & ** & : Trachelomonas & 1700 & $\star *$ \\
\hline Cyclotella & 1200 & ** & : Nitzschia & 500 & in & : Volvocales & 500 & $\mathbf{*}$ \\
\hline
\end{tabular}

* Biovolumes according to Le Cohu et al. (1991).

- Mean biovolumes (average of the genera of the same class or of the same morphology). 
Tableau 2. Biovolumes des cellules de Gymnodinium en fonction de leur diamètre.

Table 1 . Biovolumes of the cells of Gymnodinium in relation to their diameter.

\begin{tabular}{lrrrrrrrrrr}
\hline DIAMETRES $(\mu \mathrm{m})$ & 15 & 20 & 30 & 40 & 50 & 60 & 70 & 75 & 90 & 110 \\
VOLUMES $\left(\mu \mathrm{m}^{\prime}\right)$ & 590 & 1050 & 2353 & 4180 & 6450 & 9400 & 12810 & 14710 & 21180 & 47492 \\
\hline
\end{tabular}

Tableau 3. indices alimentaires (1.A.) de Rutilus rutilus.

(I.A. $=[\%$ OC $* \% \mathrm{~W}] / 100$ est compris entre 0 et 100$)$.

* N.D. = Non déterminé $\quad * P=$ Présence $(0<$ I.A. $<0,1)$.

Table 3. Indices of feeding (I.A.) of Rutilus rutilus.

(I. $A=[\%$ OC $* \%$ W] $/ 100$ is included between 0 and 100).

* N.D. $=$ Not determined $\quad * P=$ Present $(0<1 . A .<0.1)$.

\begin{tabular}{|c|c|c|c|c|c|c|c|c|c|c|c|c|c|}
\hline \multirow[b]{2}{*}{ GROUPES } & \multirow{2}{*}{$\begin{array}{l}\text { MOIS } \\
\text { CLASSES }\end{array}$} & \multicolumn{3}{|c|}{ JANVIEA } & \multicolumn{3}{|c|}{ MARS } & \multicolumn{3}{|c|}{ MAI } & \multicolumn{3}{|c|}{ IUILLET } \\
\hline & & 1 & ॥ & III & 1 & ॥ & III & 1 & II & NII & 1 & II & III \\
\hline
\end{tabular}

ZOOPLANCTON

Bosminidae
Chydoridae
Daphniidae
Calanidae
Cyclopidae
Indéterminés

PHYTOPLANCTON

\section{MACROINVERTEBRES}

Scarabaeoidae

Chironomidae

Scatopsidae

Corixidae

Dixidae

Leptoceridae

Aphidiens

Formicidae

Thysanoptères

Indéterminés

Acariens

Oligochètes

Ostracodes

AUTRES

$\begin{array}{rr}5,3 & 6 \\ 0 & \\ 2,5 & 5 \\ 0 & \\ 0 & \\ 0 & \end{array}$

P $\quad 0,1$

$\begin{array}{rll}6.9 & P & P \\ 0 & P & 0 \\ 5,5 & P & P \\ 0 & 0 & P \\ 0 & 0 & 0 \\ 0 & 0 & 9\end{array}$

$\begin{array}{rr}P & P \\ 0 & \\ P & P \\ 0 & P \\ 0 & \\ 0,8 & 0\end{array}$

$P$
0
$P$
$P$
0
0

$\begin{array}{rr}3,8 & 3,3 \\ 0 & \mathbf{P} \\ 0,1 & 3,9 \\ 0 & 0 \\ 0 & 0 \\ 32,9 & 35\end{array}$

$0,1 \quad 0,3$

$P$

0,7

$\begin{array}{rrrrrrr}0 & 0 & 0 & 0 & 0 & 0 & 0 \\ 0 & 0 & P & 2,4 & 1 & 0,3 & 0,6 \\ 0 & 0 & 0 & 0 & 0 & 0 & 0 \\ 0 & 0 & 0 & 0 & 0 & 0 & 0 \\ 0 & 0 & 0 & 0 & 0 & 0 & 0 \\ 0 & 0 & 0 & 0 & 0 & 0 & 0 \\ 0 & 0 & 0 & 0 & 0 & 0 & 0 \\ 0 & 0 & 0 & 0 & 0 & 0 & 0 \\ 0 & 0 & 0 & 0 & 0 & 0 & 0 \\ 0 & 0 & 0 & 0 & 0 & 0 & P \\ 0 & 0 & 0 & 0 & 0 & P & 0 \\ 0 & 0 & 0 & 0 & 0 & 0 & 0 \\ 0 & 0 & 0 & 0 & 0 & 0 & 0\end{array}$

$\begin{array}{rr}0 & 0 \\ 0,6 & 0 \\ 0 & 0 \\ 0 & 0 \\ 0 & 0 \\ 0 & 0 \\ 0 & 0 \\ 0 & 0 \\ 0 & 0 \\ P & 0 \\ 0 & 0 \\ 0 & 0 \\ 0 & 0\end{array}$

$\begin{array}{rr}6,1 & 37,4 \\ 0 & 0 \\ 0,6 & 5,1 \\ 0 & 0 \\ 0 & 0 \\ 0,6 & 0\end{array}$

36,1

0,5

00

$6,8 \quad 1,2$

$0 \quad 0$

$0 \quad 0$

DETRITUS

N.D. N.D. N.D.

N.D. N.D

N. N. N. N. $>0.1$

$\begin{array}{rrrr}P & 1,1 & 0 & 0 \\ 0,1 & 0 & 0 & 0,3 \\ 0,4 & 0 & 0 & 0 \\ P & 0 & 0 & 0 \\ 0 & 0 & 0 & P \\ 0 & 0 & 0 & 0,2 \\ P & 0,3 & 0 & P \\ 0 & 0 & 0 & 0,4 \\ P & 0 & 0 & 0 \\ 0 & 0 & 0 & 0,7 \\ 0 & 0 & 0 & 0,3 \\ 0 & 0 & 0,2 & 0,7 \\ 0 & 0 & 0 & P\end{array}$




\section{Résultats}

- Gardons de la classe I

Ils consomment des quantités croissantes de zooplancton (de $7,9 \%$ en janvier à $46,3 \%$ en mai) aux dépens des quantités de détritus (qui passent de $92,1 \%$ en janvier à $50,3 \%$ en mai). La contribution des Bosminidae semble toujours supérieure à celle des Daphniidae (Fig. Ia, Tableau 3).

Les proportions détritus/zooplancton restent plus ou moins stables après mai.

Les macroinvertébrés apparaissent en mars dans l'alimentation : en nombre, ils représentent la troisième source de nourriture (essentiellement des Chironomidae lors de leur émergence) mais ils ne contribuent jamais pour plus de $10 \%$ au poids total du bol alimentaire.

\section{- Gardons de la classe II}

La variation de leur régime alimentaire est similaire à celle des gardons de la classe I. Exceptée une part plus importante des détritus en mars, les proportions des différents groupes sont du même ordre de grandeur (Fig. 1b) et les items alimentaires de même nature (Tableau 3).

\section{- Gardons de la classe III}

Parmi les aliments ingérés par ces gardons, ce sont les détritus qui possèdent les valeurs d'I.A. les plus élevées (Tableau 3). La proportion des éléments détritiques est forte pendant toute la période d'étude : de $84,2 \%$ en mai à $99,2 \%$ en mars (Fig. 1c).

La part des macroinvertébrés augmente au fil des mois pour atteindre un maximum en juillet : avec $7,5 \%$ du poids du contenu intestinal, ils représentent la seconde source de nourriture. Durant les autres mois, le zooplancton reste la « catégorie de

Fig. 1. Evolution saisonnière des proportions des groupes de proies ingérées par Rutilus rutilus en fonction de leur classe de taille.

a : Gardons de la classe I.

b : Gardons de la classe II.

c : Gardons de la classe III.

Fig. 1. Seasonal changes in the proportions of groups of prey ingested by Rutilus rutilus in relation to their size -class.

a : Roach of class 1 .

b : Roach of class II,

c : Roach of class III.
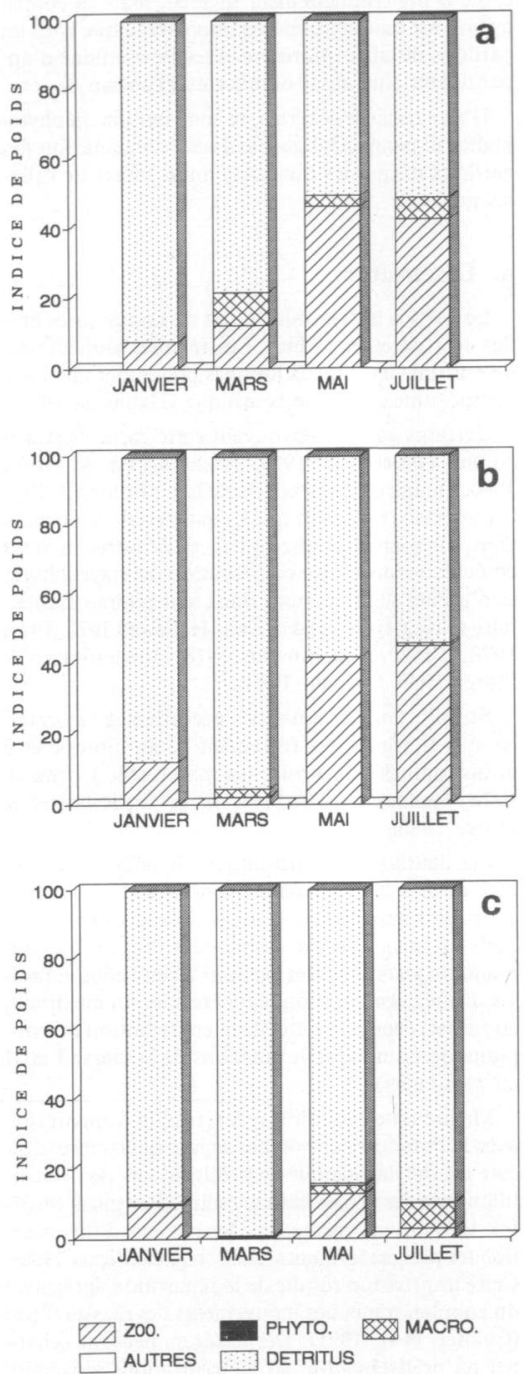
proie " préférentiellement ingérée, mais sa contribution est beaucoup moins importante que chez les gardons de taille inférieure et les Bosminidae n'apparaissent plus aussi dominants (Tableau 3).

D'une manière générale, la contribution du phytoplancton paraît négligeable dans l'alimentation des gardons malgré les quantités importantes de cellules ingérées.

\section{Discussion}

Le gardon a déjà fait l'objet de nombreuses études en rivières et en lacs. Les régimes alimentaires décrits dans les milieux lotiques presentent une forte composante d'origine benthique (Hellawell 1972).

Certains auteurs retrouvent cette caractéristique en lac (Paschalski 1958, Niederholzer \& Hofer $1980 \ldots$...) mais on ne peut généraliser ; Ponton \& Gerdeaux (1988) montrent que les gardons du lac Léman sont, au contraire, microphages. D'autres mettent en évidence une forte contribution de macrophytes et d'algues filamenteuses dans leur régime alimentaire (Cragg-Hine 1964 cité par Hellawell 1972, Prejs 1978, Prejs \& Jackowska 1978, Niederholzer \& Hofer 1980, Linfield 1980).

En fait, tous les auteurs s'accordent à reconnaître que le régime est fortement lié au biotope et à la disponibilité en proies du milieu (Mc Cormack 1970, Hellawell 1972, Prejs 1978, Niederholzer \& Hofer 1980).

Les densités zooplanctoniques du milieu (Tableau 4, Fig. 2) et les spectres alimentaires (Fig. 1), évoluent de façon similaire, à savoir une valeur minimale en mars (d'où la faible contribution du zooplancton au régime des gardons à cette époque) suivie d'une augmentation régulière avec un maximum en juillet, époque à laquelle la contribution du zooplancton est maximale (gardons de la classe I et II en particulier).

Malgré cette évolution synchrone, la comparaison avec la nourriture disponible dans le milieu reste délicate du fait du manque de précision lors de l'échantillonnage des proies dans le milieu (Ponton \& Stroffek 1987), et ce, même avec les moyens d'investigation les plus performants (Ponton \& Gerdeaux 1988). Cette imprécision résulte de la répartition agrégative du zooplancton et des mouvements des masses d'eau (Calmels et al. 1991). Des agrégats peuvent échapper au prélèvement et les concentrations moyennes
Tableau 4. Concentrations moyennes des principales familles de zooplancton et de phytoplancton dans la colonne d'eau.

Table 4. Mean concentration of the main zooplankton and phytoplankton families in the water colunn.

\begin{tabular}{ccccc} 
MOIS & JANVIER & MARS & MAl & JUILLET \\
GROUPES & $N(i / l)$ & N(i/l) & $N(i / l)$ & $N(i / l)$ \\
\hline
\end{tabular}

ZOOPLANCTON

CLADOCERES

$\begin{array}{lrrrr} & 3,23 & 0 & 4,73 & 4,06 \\ \text { Daphniidae } & 3,63 & 0,96 & 4,08 & 9,87 \\ \text { COPEPODES } & & & & \\ \begin{array}{l}\text { Calanidae } \\ \text { Cyclopidae }\end{array} & 1,81 & 0,4 & 2,32 & 2,35 \\ \text { ROTIFERES } & 0 & 0 & 0,4 & 3,43 \\ \text { Brachionidae } & 823 & 0 & 106,17 & 268,72\end{array}$

\section{PHYTOPLANCTON}

\begin{tabular}{lrrrr} 
Chrysophycae & 6040 & 632 & 860 & 1800 \\
Diatomophycae & 769896 & 177477 & 501964 & 112544 \\
Euchlorophycae & 3195 & 2541 & 8468 & 4187 \\
Zygophycae & 8920 & 6071 & 0 & 78 \\
Cryptophycae & 4339 & 415 & 2346 & 4661 \\
Euglenophycae & 1823 & 118 & 299 & 0 \\
Cyanophycae & 0 & 3615 & 3653 & 9351 \\
AUTRES & 0 & 533 & 55 & 272 \\
\hline
\end{tabular}

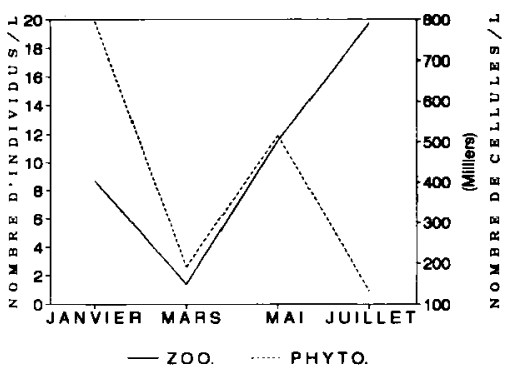

Fig. 2. Evolution saisonnière des concentrations zooplanctoniques (Copépodes + Cladocères) et phytoplanctoniques du lac de Pareloup.

Fig. 2. Seasonal changes of zooplankton (Copepoda + Cladocera) and phytoplankton concentrations in the lake of Pareloup. 
réelles pour la colonne d'eau seront sous-estimées, de même que la capture d'un nuage zooplanctonique surestimera leurs concentrations ; cela s'est probablement produit pour les Rotifères dont les concentrations observées en janvier semblent anormalement élevées (Tableau 4).

La méthode de prélèvement du phytoplancton paraît suffisante pour un tel type d'étude. Cependant, vue la contribution très « secondaire " du phytoplancton au régime des gardons étudiés, il est difficile d'établir un parallèle entre la quantité ingérée par les poissons et les variations des densités phytoplanctoniques du milieu. On constate seulement que la contribution maximale est observée dans les contenus intestinaux des gardons de la classe III en janvier (Tableau 3), mois correspondant à un développement phytoplanctonique intense (Fig. 2).

Cette étude a permis de dégager certaines tendances générales dans la nutrition des gardons du lac de Pareloup :

- Les gardons des classes I et II ont des spectres alimentaires comparables, à base de détritus et de zooplancton.

- Les gardons de taille supérieure ont une nette préférence pour les éléments détritiques, quelle que soit la saison; ce phénomène a été observé avec les gardons 5+ du lac d'Aydat en juillet 1988 (Jamet et al. 1990).

- Il existe une modification du régime alimentaire dans le temps : les détritus, abondants en janvier, diminuent assez régulièrement au profit du zooplancton ; les Cladocères (Bosminidae et daphniidae) sont alors préférentiellement ingérés aux dépens des Copépodes conformément aux résultats de la littérature (Cryer et al. 1986, Boikova 1986, Bergman 1990, Jamet et al. 1990). L'ingestion des Bosmididae semble toujours plus importante que celle des Daphniidae ; les quantités importantes de zooplancton non identifié (Tableau 3) interdisent cependant toute interprétation hâtive : en effet, les Daphniidae sont plus facilement digestibles (Gannon 1976 cité par Hyslop 1980) et peuvent donc être sousestimés. En outre, il faut également souligner l'importance de l'horaire de capture car il existe des variations notables dans les proportions des différents items zooplanctoniques ingérés (notamment parmi les Cladocères) selon l'heure de la journée (Jamet et al., op. cit.). Ce phénomène a également été observé chez Rutilus alburnö̈des hellenicus Stephanides (Daoulas 1986).

La connaissance du rythme de prise alimentaire prend ici toute son importance. L'analyse des contenus intestinaux doit être réalisée aussitôt après la prise de nourriture de sorte que les proies contenues dans le tube digestif soient dans un état de dégradation minimum. Lors de prises de nourriture plusieurs fois par jour comme c'est le cas pour les gardons de Pareloup (Arias et al., sous presse), l'analyse des tubes doit être faite après chaque phase d'alimentation. Ainsi, une quantification journalière des différentes catégories de proies devient possible.

- L'absence de Copépodes dans le régime de Rutilus rutilus peut s'expliquer par le fait que les Copépodes possèdent des mécanismes de détection des poissons et échappent ainsi assez facilement aux filtreurs par déplacement (Flemminger \& Clutter 1965).

- Les macroinvertébrés, le plus souvent représentés par les Diptères Chironomidae, entrent dans la composition du régime alimentaire à partir de mars, mais leur contribution pondérale est secondaire.

- Des matières allochtones peuvent être ingérées par les gardons : grains de pollen, spores de champignons, macroinvertébrés exogènes (Scarabaeoïdae, Scatopsidae, Aphidiens, Formicidae...) mais de façon moins significative.

- Le phytoplancton (essentiellement Diatomophycées) semble ingéré aléatoirement, mais sa contribution pondérale est faible, voire négligeable. Reste à préciser son importance énergétique et sa contribution réelle à la croissance des animaux.

Comparativement aux résultats de la littérature, le régime décrit pour les gardons de Pareloup présente certaines variantes. En effet, on ne trouve pas de « régime type » pour le gardon : la composition de son alimentation dépend de la nourriture présente et d'autres facteurs écologiques.

Le spectre assez large du gardon et sa capacité à se nourrir de détritus, pour les sujets les plus âgés, est une adaptation liée à son processus spécifique de digestion (Persson 1983a).

Les gardons s'adaptent donc facilement à différents types de nourriture et ne manifestent pas d'exigences particulières dans ce domaine. 
Les résultats montrent enfin que les gardons ne connaissent pas de périodes de jeûne hivernal très prononcées comme cela est parfois décrit dans d'autres écosystèmes (Hartley 1947). Nos conclusions, sur la période étudiée, rejoignent plutôt celles de Cragg-Hine (1964, in Hellawell 1972) qui signale que $R$. rutilus se nourrit activement toute l'année, soulignant toutefois que les quantités de nourriture consommées en hiver sont inférieures à celles ingérées au printemps et en été.

\section{Travaux cités}

Albertine-Berhaut J. 1973. - Biologie des stades juvéniles de Téléostéens Mugilidae Mugil auratus Risso 1810, Mugil cupito Cuvier 1829 et Mugil saliens Risso 1810. Aquaculture, 2 : 251-266.

Arias-Gonzalez J.E., Richeux C. \& Tourenq J.N. Sous presse - Evaluation de la ration journalière et de la consommation de nourriture pour une population de gardons (Rutilus rutilus) du lac de Pareloup (Aveyron, France). Cybium.

Bergman E. 1990. - Effects of roach Rutilus rutilus on two percids, Perca fluviatilis and Gymnocephalus cernua : importance of species interactions for diet shifts. Oikos, 57 : 241-249.

Boikowa O.S. 1986. - Feeding of fish in Lake Glubokoe. Hydrobiologia, $141: 95-111$.

Bottrel H.H., Duncan A. \& Gliwicz M. 1976. - A review of some problems in zooplankton production studies. Norw. J. Zool., $24: 419.456$.

Calmels P. \& Salençon M.J. 1991. - Techniques de marquages pour l'étude des circulations des masses d'eau dans les lacs. Application à la retenue de Pareloup. Hydroécol. Appl., 1 (3) : 27-42.

Cragg-Hine D. 1964. - The biology of the coarse fish of Willow Brook, Northamptonshire. Ph. D. thesis, University of Liverpool (Citè par Hellawell 1972).

Cryer M., Peirson G. \& Thownsend C.R. 1986. - Reciprocal interactions between roach, Rutilus rutilus, and zooplankton in small lakes. Prey dynamics and fish growth and recruitment. Limnol. Oceanogr., 31 (5) : 1022-1038.

Daoulas C. 1986. - Diurnal feeding pattern of Rutilus alburnoides hellenicus Stephanides (Pisces, Cyprinidae) in Lake Trichonis, Greece. Acta hydrobiol., $28: 227-235$.

Dumont H.J., Van de Velde J. \& Dumont S. 1975. - The dry weight estimate of biomass in a selection of Cladocera, Copepoda and Rotifera from the plankton, periphyton and benthos of continental waters. Oecologia, $19: 79-97$.

Flemminger A. \& Clutter R.I. 1965. - Avoidance of towed nets by zooplankton. Limnol. Oceanogr., $10: 96-104$.

Gannon J.E. 1976. - The effects of differential digestion rates on zooplankton by Alewife. Alosa pseudoharengus on determination of selective feeding. Trans, Am. Fish. Soc. 105, 89-95. (Cité par Hyslop 1980).

Hartley P.H.T. 1947. - The natural history of some British freshwater fishes. Proc, zool. Soc. Lond., 117 : 129.206.

Hellawell J.M. 1972, - The growth, reproduction and food of the roach (Rutilus rutilus (L.)), of the River Lugg, Herefordshire. J. Fish. Biol., 4 : 469-486.
Hynes B.N. 1950. - The food of freshwater sticklebacks (Gasteroteus aculeat us and Pygosteus pongitius) with a review of methods used in studies of the food of fishes. J. anim. Ecol., $19: 36-58$.

Hyslop E.J. 1980. - Stomach contents analysis - a review of methods and their application. J. Fish. Biol., $17: 4 ! 1-429$.

Jamet J.L., Gres P., Lair N. \& Lasserre G. 1990. - Diel feeding cycle of roach (Rutilus rutilus (L.)) in eutrophic Lake Aydat (Massif Central, France). Arch. Hydrobiol, 118 (3) : $371-382$.

Lauzanne L. 1976. - Régimes alimentaires et relations trophiques des poissons du lac Tchad. Cah. ORSTOM, sér. Hydrobiol., $10: 267-310$.

Le Cohu R., Comoy N., Guitard J. \& Brabet J. 1991 . - Périodicité du phytoplancton dans un réservoir de moyenne profondeur : le lac de Pareloup (Massif Central, France), un exemple de succession cyclique. Annls Limnol., 27 (3) : 197-214.

Linfield R.S.J. 1980. - Ecological changes in a lake fishery and their effects on a stunted roach (Rutilus rutilus) population. J. Fish. Biol., $16: 123-144$.

Mc Cormack J.C. 1970. - Observations of the food of perch (Perca fluviatilis) in Windermere. J. anim. Ecol., 39 : 255-267.

Niederholzer R. \& Hofer R. 1980. - The feeding of roach (Rutihus rutilus (L.)) and rudd (Scardinius erythrophthalmus (L.)). I. Studies on natural populations. Ekol. pol., 28 (1) : 45-59.

Northcote T.G. 1988. - Fish in the structure and function of freshwater ecosystems : A « Top-down 》 view. Can. J. Fish. Aquat. Sci, $45: 361-379$.

Palomares M.L. \& Pauly D. 1989. - A multiple regression model for predicting the food consumption of marine fish populations. Aust. J. mar. Freshwater Res., 40:259-273.

Paschalski J. 1958. - The feed of Rutilus rutilus (L.) and Chondrostoma nasus (L.) in the Roznow Dam Water Reservoir. Pol. Arch. Hydrobiol., 5 : 55-64.

Persson L. 1983a. - Food consumption and the significance of detritus and algae to intraspecific competition in roach $R$ utilus rutilus in a shallow eutrophic lake. Oikos, $41: 118-125$.

Ponton D. \& Gerdeaux D. 1988. - Quelques aspects de deux poissons planctonophages du lac Léman : le corégone (Coregonus schinziil palea Cuv, et Val.) et le gardon (Rutilus rutilus (L.)). Bull. Fr. Pêche Piscic., 308 : 11-23.

Ponton D. \& Stroffek S. 1987. - Régime alimentaire des alevins de gardons (Rutilus rutilus (L.)) dans un port du lac Léman. Comparaison avec la nourriture disponible. Schweiz. Z. Hydrol., 49 (3) : 329-342.

Prejs A. \& Jackowska H. 1978. - Lake macrophytes as the food of roach (Ruilus rutilus (L.)) and rudd (Scardinitus erythrophthalmus (L.)). I. Species composition and dominance relations in the lake and food. Ekol. pol., 26 (3) : 429-438.

Prejs A. 1978. - Lake macrophytes as the food of roach (Rutilus rutilus ( $\mathrm{L}$.)) and rudd (Scardinius erythrophtalmus (L.)). II. Daily intake of macrophyte food in relation to body size of fish. Ekol. pol., $26: 537-553$.

Reynolds C.S. 1984. - The ecology of freshwater phytoplankton. Cambridge University Press : $384 p$.

Straskraba M. 1965. - The effect of fish on the number of invertebrates in ponds and streams. Mitt. int. Ver. Limnol., 13 : 106-127,

Windell J.T. 1968. - Food analyses and rates of digestion. In Ricker W.E. (ed.), Methods for assessment of fish production in fresh waters. Blackwell Scientific Publications, Owford and Edinburght, 197-203. 